\title{
Quantum Computing Using Dissipation to Remain in a Decoherence-Free Subspace
}

\author{
Almut Beige, ${ }^{1}$ Daniel Braun, ${ }^{2}$ Ben Tregenna, ${ }^{1}$ and Peter L. Knight ${ }^{1}$ \\ ${ }^{1}$ Optics Section, Blackett Laboratory, Imperial College London, London SW7 2BZ, England \\ ${ }^{2}$ FB7, Universität-GHS Essen, 45117 Essen, Germany \\ (Received 16 November 1999; revised manuscript received 10 April 2000)
}

\begin{abstract}
We propose a new approach to the implementation of quantum gates in which decoherence during the gate operations is strongly reduced. This is achieved by making use of an environment induced quantum Zeno effect that confines the dynamics effectively to a decoherence-free subspace.
\end{abstract}

PACS numbers: 03.67.Lx, 42.50.Lc

Quantum computing has attracted much interest since it became clear that quantum computers are in principle able to solve hard computational problems more efficiently than present classical computers [1-3]. The main obstacle inhibiting realizations arises from the difficulty of isolating a quantum mechanical system from its environment. This leads to decoherence and the loss of information stored in the system, which limits for instance factoring to small numbers [4]. Schemes have been proposed to correct for errors induced by decoherence and other imperfections [5]. Alternatively, the use of decoherence-free subspaces [6-9] has been proposed for which the dependence on error correction codes may be much reduced. Nevertheless, the error rate of each operation must not exceed $10^{-5}$ if quantum computers are ever to work fault tolerantly [10].

In contrast to the widely held folk belief that decoherence is to be avoided, we show here that dissipation can be used to implement nearly decoherence-free quantum gates with a success rate which can, at least in principle, be arbitrarily close to unity. The main requirement for this to work is the existence of a decoherence-free subspace (DFS) in the system under consideration. States in the DFS will be called decoherence-free (DF) states. Examples of DFS are known $[9,11]$, but until now, it was not known how to manipulate states within a DFS in general [12].

In this Letter we propose a concrete example of a DFS whose states can be used to obtain DF qubits for quantum computing. In contrast to earlier proposals, we assume that all other states couple strongly to the environment. A state with no overlap with DF states should (nearly immediately) lead to dissipation. We show that we can interpret the effect of the environment on the system as that of rapidly repeated measurements of whether the system is DF or not. This effect, which we call an environment induced quantum Zeno effect [13], leads to the fact that a weak interaction changes only the state of the system inside the DFS. This allows for a wide range of new possibilities to perform DF gate operations between the qubits. As an example we describe a CNOT operation between two qubits that is almost DF yet rather simple: A single laser pulse suffices. We will show that the system proposed fulfills all criteria for a quantum computer proposed by DiVincenzo [14].
The system we propose consists of $N$ identical threelevel atoms with a $\Lambda$ configuration. We denote the split ground states of atom $i$ by $|0\rangle_{i}$ and $|1\rangle_{i}$, and the excited state by $|2\rangle_{i}$. The atoms are assumed to be stored in a line, which can be for instance in a linear ion trap, an optical lattice, or on top of a wire on an atom chip [15]. To realize a gate operation between two neighboring atoms (denoted by $i=1$ and $i=2$ in the following), requires to move them into a cavity, as shown in Fig. 1. This can be done by moving the lattice or by applying an electric field, respectively. We assume that only the atomic 1-2 transition is in resonance with a single resonator mode. For simplicity the coupling constants of both atoms to the cavity field mode are taken to be the same, $g_{1}=g_{2} \equiv g$, but this is not crucial to our analysis.

The environment consists of a continuum of electromagnetic field modes surrounding the atoms and the cavity. This gives rise to decoherence in two different ways: First, individual spontaneous emission of the atoms outside the cavity can take place with a rate $\Gamma$. For atoms inside the cavity this rate can be decreased to below its free-space value and will be denoted by $\Gamma_{\text {cav }}$. In addition, the resonant field mode inside the cavity couples to the outside, given nonideal mirrors. A photon inside the resonator leaks out through the cavity mirrors with a rate $\kappa$.

To describe the time evolution of the system and to find a simple criterion for a DFS we will make use of a quantum jump description [16]. This method gives the time evolution under the condition that no photon is emitted, as well as the probability for no photon emission,

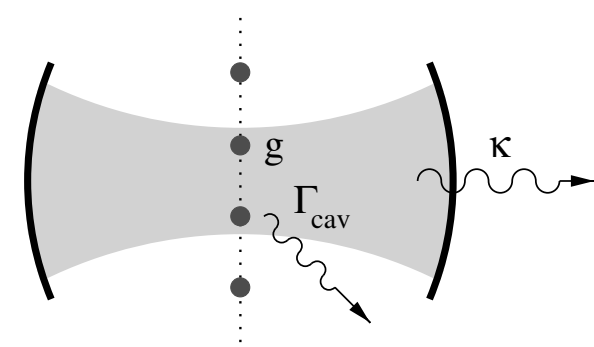

FIG. 1. Schematic view of the system. To perform a gate operation two three-level atoms are moved to fixed positions inside a cavity. 
$P_{0}(t, \psi)$, where $|\psi\rangle$ is the state of the system at time $t=0$. The system dynamics is described by a non-Hermitian Hamiltonian $H_{\text {cond }}$ that incorporates the coupling to the environment. It can be derived rigorously from the full Hamiltonian. Because of the non-Hermiticity, the norm of the state vector

$$
\left|\psi^{0}(t)\right\rangle=e^{-i H_{\text {cond }} t / \hbar}|\psi\rangle
$$

decreases with time. The probability $P_{0}$ to observe no photon up to time $t$ by a broadband detector of unit efficiency is given by the squared norm

$$
P_{0}(t, \psi)=\left\langle\psi^{0}(t) \mid \psi^{0}(t)\right\rangle .
$$

The negative derivative of $P_{0}$ at time $t=0$ gives the probability density for an immediate photon emission from state $|\psi\rangle$ and equals

$$
I(\psi)=\frac{i}{\hbar}\left\langle\psi\left|H_{\text {cond }}-H_{\text {cond }}^{\dagger}\right| \psi\right\rangle .
$$

If no photon is observed, the state of the system at time $t$ is the state (1) normalized to unity.

In the following $b$ denotes the annihilation operator for one photon in the cavity mode. If we choose the interaction picture in a way that the atoms and the cavity mode plus environment are considered as the free system one finds in a similar way as in Ref. [17] that the conditional Hamiltonian equals

$$
\begin{aligned}
H_{\text {cond }}= & i \hbar g \sum_{i=1}^{2}\left[b|2\rangle_{i i}\langle 1|-\text { H.c. }\right] \\
& -i \hbar \Gamma_{\text {cav }} \sum_{i=1}^{2}|2\rangle_{i i}\langle 2| \\
& -i \hbar \Gamma \sum_{i=3}^{N}|2\rangle_{i i}\langle 2|-i \hbar \kappa b^{\dagger} b .
\end{aligned}
$$

According to the above, a simple criterion for a DF state of the atoms and the cavity field mode is: No photon should be emitted, either by spontaneous emission or by leakage of a photon through the cavity mirrors. A state $|\psi\rangle$ belongs to the DFS if and only if

$$
P_{0}(t, \psi) \equiv 1 \quad \forall t \geq 0 .
$$

As can be seen from Eq. (3) no photon emission is possible if the atoms are all in a ground state and the cavity field is empty. The interaction of the system with the environment is effectively switched off [8]. In addition, there is no energy in the system which can be emitted in the form of a photon. The $2^{N}$ DF ground states of the system are therefore ideally suited as the $N$ DF qubit memory for the quantum computer [18]. The $i$ th qubit is formed by the two ground states $|0\rangle_{i}$ and $|1\rangle_{i}$ of atom $i$ while there is no photon in the resonator mode.

In addition to these states we obtain more DF states if we neglect spontaneous emission by the atoms inside the cavity. These states should only become populated during gate operations and allow for nearly DF gates. For $\Gamma_{\text {cav }}=0$ given Eq. (3) no photon emission can also take place if a state $|2\rangle_{i}(i=1,2)$ of the atoms inside the cavity is excited. However, this is not yet sufficient. The cavity mode must never become populated, i.e., the system's own time evolution must not drive states out of the DFS [8]. In the following we denote by $|n \varphi\rangle \equiv|n\rangle \otimes|\varphi\rangle$ a state with $n$ photons in the cavity and the atoms in state $|\varphi\rangle$. A state $|0 \varphi\rangle$ is DF if all matrix elements of the form $\left\langle n \varphi^{\prime}\left|H_{\text {cond }}\right| 0 \varphi\right\rangle$ vanish for $n \neq 0$ and arbitrary $\varphi^{\prime}$. This is the case if and only if

$$
J_{-}|\varphi\rangle \equiv \sum_{i=1,2}|1\rangle_{i i}\langle 2 \mid \varphi\rangle=0 .
$$

Besides the superpositions of the atomic ground states the atoms inside the cavity can also be in a superposition with the trapped state $|a\rangle \equiv\left(|1\rangle_{1}|2\rangle_{2}-|2\rangle_{1}|1\rangle_{2}\right) / \sqrt{2}$, a maximally entangled state of the two atoms $[17,19]$.

For $\Gamma_{\text {cav }}=0$ one finds from Eqs. (4) and (6) that $H_{\text {cond }}|\psi\rangle=0$. Without an additional interaction a DF state does not change in time. To manipulate the states inside the DFS a weak interaction can be used. But before we discuss the effect of this interaction we need to study the effect of the environment on the system in more detail. Let us define the time $\Delta T$ as the minimum time in which a system in an arbitrary state outside the DFS definitely emits a photon. Then we can interpret the observation of the free radiation field outside the cavity over a time interval $\Delta T$ as a measurement of whether the system is in a DF state or not. The outcome of the measurement is indicated by the emission of a photon (no DF state), or its absence (DF state).

Here, the cavity field interacts continuously with its environment and the system behaves like a system under continuous observation, e.g., the time between two consecutive measurements is zero. In such a case the quantum Zeno effect [13] can be used to predict the time evolution of the system in the presence of a weak interaction which tries to change the state of the system. The quantum Zeno effect is a consequence of the projection postulate for ideal measurements and suggests that any process that would lead out of the DFS is "frozen" by the measurements, which always project the system back into a DF state. In this way the interaction with the environment protects the system against dissipation. On the other hand, the dynamics within the DFS is insensitive to the measurements and takes place almost unmodified.

In the following $H_{\text {cond }}$ describes the conditional time evolution of the system in the presence of an interaction. As long as the interaction is weak enough, the effect of the environment on the system can still be interpreted to a good approximation as rapidly repeated measurements. Therefore the time development operator over the small time interval $\Delta T$ is given by $\mathbb{P}_{\mathrm{DFS}} U_{\text {cond }}(\Delta T, 0) \mathbb{P}_{\mathrm{DFS}}$, where $\mathbb{P}_{\text {DFS }}$ is the projector on the decoherence-free subspace. This leads to the effective Hamiltonian

$$
H_{\mathrm{eff}}=\mathbb{P}_{\mathrm{DFS}} H_{\mathrm{cond}} \mathbb{P}_{\mathrm{DFS}},
$$


which has a very different effect compared to atoms in free space.

As an example and to show how to realize a CNOT gate we consider a weak laser pulse applied to the atoms inside the cavity only. The atoms should be spatially well separated so that the laser pulses can be applied to each atom individually. The complex Rabi frequencies for the $j-2$ transition $(j=0,1)$ of atom $i(i=1,2)$ are denoted by $\Omega_{j}^{(i)}$. If a laser irradiates the atoms the Hamiltonian

$$
H_{\text {laser I }}=\frac{\hbar}{2} \sum_{i=1}^{2} \sum_{j=0}^{1}\left[\Omega_{j}^{(i)}|j\rangle_{i i}\langle 2|+\text { H.c. }\right]
$$

has to be added to the right-hand side of Eq. (4). The Rabi frequencies $\Omega_{j}^{(i)}$ set the time scale on which the states of the atoms are changed due to the laser. This time must be much longer than the measurement time $\Delta T$ which is of the order of $1 / \kappa$ and $\kappa / g^{2}$. In addition spontaneous emission by the atoms has to be negligible during the gate operation which leads to the condition

$$
\Gamma_{\text {cav }} \ll\left|\Omega_{j}^{(i)}\right| \ll \kappa \text { and } g^{2} / \kappa .
$$

A CNOT gate performs a unitary operation in which the value of one qubit is changed if and only if the control bit is in state $|1\rangle$. We choose the first qubit as the control bit which means that the gate should exchange the states $|010\rangle$ and $|011\rangle$, while the states $|000\rangle$ and $|001\rangle$ remain unchanged. (Here the state $\left|0 j_{1} j_{2}\right\rangle$ describes a system with no photons in the cavity while the atoms are in state $\left|j_{1}\right\rangle_{1}\left|j_{2}\right\rangle_{2}$ ). Eqs. (4), (7), and (8) and the choices

$$
\begin{gathered}
\Omega_{1}^{(1)}-\Omega_{1}^{(2)}=\sqrt{2} \Omega, \quad \Omega_{0}^{(2)}=\sqrt{2} \Omega, \\
\text { and } \Omega_{0}^{(1)}=0
\end{gathered}
$$

for the Rabi frequencies lead together with $\Gamma_{\text {cav }}=0$ to the effective Hamiltonian

$H_{\mathrm{eff}}=\frac{\hbar}{2}[\Omega(|010\rangle\langle 0 \mathrm{a}|-| 0 \mathrm{a}\rangle\langle 011|)+$ H.c. $]$.

A single laser pulse of length $T=\sqrt{2} \pi /|\Omega|$ therefore yields the desired time evolution operator for the CNOT,

$$
U_{\text {eff }}(T, 0)=|010\rangle\langle 011|+\text { H.c. }
$$

Here $H_{\text {eff }}$ is Hermitian and due to Eq. (2) the probability for a photon emission during the laser pulse is, within the approximations made, not possible.

It might be helpful to illustrate the mechanism which confines the dynamics to the DFS in more detail. The time evolution of the system under the condition of no photon emission is given by the conditional Hamiltonian $H_{\text {cond }}+H_{\text {laser I }}$. The full equations of motion resulting from Eq. (1) reveal that only the amplitudes of DF states change slowly in time, on a time scale proportional to $1 /|\Omega|$. If the system is initially in a DF state the laser pulse excites the states outside the DFS. Then the excitation is transferred with a rate proportional $g$ into states in which the cavity mode is populated. Those states are immediately emptied by one of the following two mechanisms: One possibility is that a photon leaks out through the cavity mirrors. But, as long as the population of the cavity field is small, the leakage of a photon through the cavity mirrors is unlikely to take place. With a much higher probability the excitation of the cavity field vanishes during the conditional time evolution due to the term $-i \hbar \kappa b^{\dagger} b$ in the conditional Hamiltonian in Eq. (4). No population can accumulate outside the DFS.

We also derived the time evolution of the DF states by adiabatically eliminating the amplitudes of all non-DF states. This is possible due to the frequency scale separation (9). To lowest order in $\Omega / \kappa$ and $\Omega \kappa / g^{2}$ we recover Eq. (12). The more precise result including the next higher order allows for an optimization of the gate operations [20].

If one assumes $\Gamma_{\text {cav }} \neq 0$ the state $|0 a\rangle$ does not correspond to a DF state and a photon may be emitted during the gate operation. In addition finite parameters of $g$ and $\kappa$ may lead to the leakage of photons through the cavity mirrors. These effects have been taken into account in Fig. 2 which results from a numerical solution of the Schrödinger equation (1) and shows the probability for no photon emission during a single CNOT operation. Here the initial state $|010\rangle$ was chosen. The figure confirms that for vanishing spontaneous emission the probability of success becomes arbitrarily close to unity if $|\Omega|$ is made small. For finite $\Gamma_{\text {cav }}$, spontaneous emission is the limiting factor due to the increasing duration of the operation for small $|\Omega|$. If no photon is emitted during the gate operation, the fidelity of the state at the end of the pulse compared to a state expected for an ideal CNOT operation is very high. For the parameters used in Fig. 2 the final amplitude of the desired state $|011\rangle$ is always higher than $98 \%$ [20].

Finally, we point out that there exists a very simple scheme in which the transition between a DF state and non-DF states is also strongly inhibited, a three-level atom

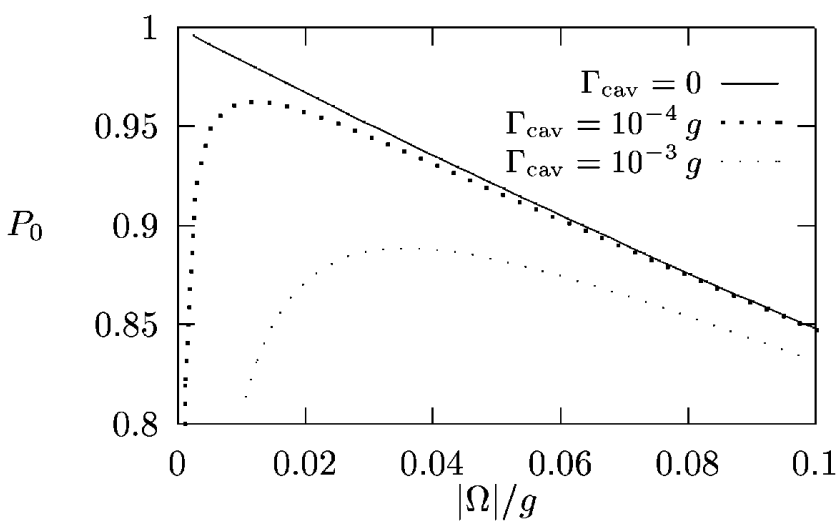

FIG. 2. The probability for no photon emission during a CNOT operation as a function of the Rabi frequency $\Omega$ for $\Omega_{0}^{(2)}=$ $\sqrt{2} \Omega, \Omega_{1}^{(1)}=-\Omega_{1}^{(2)}=\Omega / \sqrt{2}, \kappa=g$, and different values of $\Gamma_{\text {cav }}$. The system is initially in the state $|010\rangle$. 
with a $\mathrm{V}$ configuration. One transition between the ground state and a metastable state of the atom is driven by a very weak laser field with Rabi frequency $\Omega_{\mathrm{w}}$, while a laser with a very high Rabi frequency $\Omega_{\mathrm{s}}$ couples the ground state to a level with a high decay rate $\Gamma_{\mathrm{s}}$. In this scheme the metastable state corresponds to the DFS, while $\left|\Omega_{\mathrm{s}}\right|$ plays the role of the coupling constant $g$ and $\Gamma_{\mathrm{s}}$ the role of $\kappa$. Once in a metastable state the atom remains there for a long time proportional to $\left(\left|\Omega_{\mathrm{s}}\right| /\left|\Omega_{\mathrm{w}}\right|\right)^{2}$ [21]. This is known as a macroscopic dark period and the scheme has been used to test the quantum Zeno effect experimentally [22]. Equally, we expect for the scheme proposed here that the mean time before photon emission is proportional to $(g /|\Omega|)^{2}$ and is much longer than the gate duration which is proportional to $g /|\Omega|$. This is shown explicitly in Ref. [20] and encourages us to believe that our proposal is experimentally feasible. Because of the correspondence of these schemes we could also describe our proposal as "quantum computing in a dark period."

Our system also fulfills the remaining criteria for a quantum computer [14]. The single qubit rotation, which together with the CNOT forms a "universal" set of quantum gates, can be performed with the help of an adiabatic population transfer [23] - a technique which has been realized with high accuracy in experiments [24]. It requires two laser pulses and the laser fields couple to the 0-2 and 1-2 transitions, respectively, with the same large detuning [20]. The readout of the information stored in the qubits can be realized with an electron shelving technique [25]. The system is scalable, with well-characterized qubits, can be prepared in a defined initial state, and the relevant decoherence time is much longer than the gate operation time if condition (9) can be achieved. In summary, we have made a proposal for quantum computing using dissipation. Why the system remains in a DFS can be understood in terms of the quantum Zeno effect.

We thank F. Haake, W. Lange, R. Laflamme, D. A. Lidar, M. B. Plenio, and T. Wellens for discussions. Part of this work was done at the ESF-Newton Institute Conference in Cambridge, partially supported by the ESF. This work was also supported by the A.v. Humboldt Foundation, by the European Union, by the UK Engineering and Physical Sciences Research Council, and by the Sonderforschungsbereich 237 "Unordnung und große Fluktuationen."
[1] D. Deutsch, Proc. R. Soc. London A 400, 97 (1985); 425, 73 (1989).

[2] P.W. Shor, in Proceedings of the 35th Annual Symposium on Foundations of Computer Science (IEEE Computer Society Press, Santa Fe, NM, 1994).

[3] L. K. Grover, Phys. Rev. Lett. 79, 325 (1997).

[4] M. B. Plenio and P. L. Knight, Proc. R. Soc. London A 453, 2017 (1997); Phys. Rev. A 53, 2986 (1996).

[5] P. W. Shor, Phys. Rev. A 52, R2493 (1995); A. M. Steane, Proc. R. Soc. London A 452, 2251 (1996); A. R. Calderbank and P. W. Shor, Phys. Rev. A 54, 1098 (1996).

[6] G. M. Palma, K.-A. Suominen, and A. K. Ekert, Proc. R. Soc. London A 452, 567 (1996).

[7] P. Zanardi and M. Rasetti, Phys. Rev. Lett. 79, 3306 (1997).

[8] D. A. Lidar, I. L. Chuang, and K. B. Whaley, Phys. Rev. Lett. 81, 2594 (1998).

[9] L. M. Duan and G. C. Guo, Phys. Rev. A 58, 3491 (1998).

[10] J. Preskill, Proc. R. Soc. London A 454, 385 (1998); A. M. Steane, quant-ph/9809054.

[11] P. Zanardi and F. Rossi, Phys. Rev. B 59, 8170 (1999).

[12] However, see D. Bacon, J. Kempe, D. A. Lidar, and K. B. Whaley, quant-ph/9908064; J. Kempe, D. Bacon, D. A. Lidar, and K. B. Whaley, quant-ph/0004064.

[13] B. Misra and E. C. G. Sudarshan, J. Math. Phys. 18, 756 (1977).

[14] D. P. DiVincenzo, quant-ph/0002077.

[15] R. Folman, P. Krüger, D. Cassettari, B. Hessmo, T. Maier, and J. Schmiedmayer, Phys. Rev. Lett. 84, 4749 (2000).

[16] G.C. Hegerfeldt and D. G. Sondermann, Quantum Semiclass. Opt. 8, 121 (1996). For a recent review see M. B. Plenio and P. L. Knight, Rev. Mod. Phys. 70, 101 (1998), and references therein.

[17] M. B. Plenio, S.F. Huelga, A. Beige, and P.L. Knight, Phys. Rev. A 59, 2468 (1999).

[18] T. Pellizzari, S. A. Gardiner, J. I. Cirac, and P. Zoller, Phys. Rev. Lett. 75, 3788 (1995).

[19] P. M. Radmore and P. L. Knight, J. Phys. B 15, 561 (1982); G. M. Meyer and G. Yeoman, Phys. Rev. Lett. 79, 2650 (1997); G. J. Yang, O. Zobay, and P. Meystre, Phys. Rev. A 59, 4012 (1999).

[20] B. Tregenna, A. Beige, and P. L. Knight (to be published).

[21] A. Beige and G. C. Hegerfeld, J. Phys. A 30, 1323 (1997).

[22] W. M. Itano, D. J. Heinzen, J. J. Bollinger, and D. J. Wineland, Phys. Rev. A 41, 2295 (1990).

[23] N. V. Vitanov and S. Stenholm, Phys. Rev. A 55, 648 (1997).

[24] K. Bergmann, H. Theuer, and B. W. Shore, Rev. Mod. Phys. 70, 1003 (1998).

[25] A. Beige and G. C. Hegerfeldt, J. Mod. Opt. 44, 345 (1997). 